\title{
Kulturelle Unterschiede bezüglich der depressiven Störung und Bindungsorientierung
}

\author{
Elif Gündüz - Barbara H. Juen · Willi Geser · Ahmet Ayer · Eberhard A. Deisenhammer
}

Eingegangen: 16. Januar 2018 / Angenommen: 19. März 2018 / Online publiziert: 10. April 2018

(c) Der/die Autor(en) 2018.

\section{Zusammenfassung}

Grundlagen Bindung ist ein kulturübergreifender, psychologischer Parameter, der durch Kindheitserfahrungen geformt wird. Obwohl das Bindungskonstrukt zur Stabilität neigt, können verschiedene, auch kulturelle Einflussfaktoren im Laufe des Lebens zu Veränderungen der Bindungsqualität führen. Das Beispiel der depressiven Störung im Erwachsenenalter macht die Bedeutsamkeit des kulturellen Hintergrundes anhand der klinisch differenten Manifestation der Symptome deutlich.

Methodik In der vorliegenden Studie wurden interund intrakulturelle Unterschiede hinsichtlich der Bindungsqualität der drei Untersuchungsgruppen Türken im Heimatland, türkische Migranten und Österreicher $(N=297)$ mittels des Selbstbeurteilungsfragebogens RSQ untersucht. Innerhalb aller drei Gruppen wurde jeweils eine gesunde Vergleichs- $(N=153)$ sowie eine aktuell in einer depressiven Episode befindliche Patientengruppe $(N=144)$, die entweder stationär oder ambulant behandelt wurde, untersucht.

Aus Gründen der besseren Lesbarkeit wird auf die gleichzeitige Verwendung männlicher und weiblicher Sprachformen verzichtet. Sämtliche Personenbezeichnungen gelten gleichwohl für beiderlei Geschlecht.

E. Gündüz $(\bowtie) \cdot$ B. H. Juen $\cdot$ W. Geser

Institut für Psychologie, Leopold-Franzens-Universität Innsbruck, Innsbruck, Österreich

elif.g@gmx.at

A. Ayer

Manisa Ruh Sağlığı ve Hastalıkları Hastanesi, Manisa, Türkei

E. A. Deisenhammer

Universitätsklinik für Psychiatrie 1, Medizinische Universität Innsbruck, Innsbruck, Österreich
Fragestellung Moderiert die Kultur einen Zusammenhang zwischen der depressiven Erkrankung und der Bindungsorientierung im Erwachsenenalter?

Ergebnisse Interkulturell betrachtet waren die Vergleichs- im Gegensatz zu den Patientengruppen in allen Bindungsparametern als sicherer gebunden einzustufen. Während die österreichische Vergleichsgruppe dabei die sicherste Bindungsorientierung zeigte, wiesen die österreichischen Patienten, gefolgt von den Migranten, die höchste Unsicherheit auf. Im Vergleich der depressiven und gesunden Türken waren hingegen keine signifikanten Unterschiede feststellbar.

Schlussfolgerung Die Kultur zeigt somit signifikante Effekte in Bezug auf die depressive Störung und die Bindungsdimensionen.

Schlüsselwörter Kultur · Bindungsqualität · Depression · Individualismus · Kollektivismus

\section{Cultural differences concerning depressive disorders and attachment dimensions}

\section{Summary}

Background Attachment is a psychological parameter across cultures and is influenced by childhood experiences. Though the construct of attachment tends to certain stability, different influences, like culture, can change the quality of attachment during lifetime. As can be seen from the example of depression in adulthood the clinical symptoms of this disorder may be different due to the cultural background.

Methods The present study focuses on inter- and intracultural differences concerning the attachment qualities of Turkish people in the homeland, Turkish migrants in Austria and Austrians $(N=297)$ by using the self-assessment questionnaire RSQ. Each ethnic group consisted of a healthy control group $(N=153)$ as well as a patient group currently suffering from 
a depressive episode $(N=144)$ and were getting an in or outpatient treatment.

Objective The hypothesis tested was whether culture moderates a correlation between depressive disorders and attachment abilities.

Results There were significant differences in attachment ability between cultures whereby controls presented significantly more secure attachment ability in all parameters compared to patients. While the Austrian control group showed the most secure attachment orientation, the Austrian patients had the highest level of insecurity, followed by the migrant group. However, the comparison of healthy and depressive Turks revealed no significant differences.

Conclusion Thus, culture shows significant effects concerning depressive disorders and attachment dimensions.

Keywords Culture - Attachment quality · Depressive disorder · Individualism · Collectivism

\section{Einleitung}

Die Bindungstheorie von Bowlby [1] sieht Bindung als eine biopsychische Motivation, denn erst durch das Herstellen und Aufrechterhalten emotionaler Bande zu einer Bindungsperson wird das Überleben des Menschen gesichert [2]. Demzufolge fungieren die Eltern als sicherer Hafen, von dem aus das Explorationsverhalten des Säuglings gefördert und gestärkt werden kann [3]. Neben dem Vorhandensein einer sogenannten zentralen Bindungsperson [4] können jedoch auch Determinanten wie beispielsweise die Feinfühligkeit der Mutter [5], das kindliche Temperament [6] sowie sozio-ökonomische Faktoren [7, 8] die Bindungsqualität beeinflussen. Auch ist es von Bedeutung, von welcher Kultur die Interaktion zwischen Bindungsperson und Kind geprägt wird [9], da diese Auswirkungen auf die Familiensysteme und somit auf den Erziehungsstil hat [10-12]. Auf diesen Erkenntnissen basierend konnten drei unterschiedliche Verhaltensmuster und daraus resultierend Bindungsstile identifiziert werden [13]: die sichere, die unsicher-vermeidende und die unsicher-ambivalente Bindung. 1986 wurde von Main und Solomon zusätzlich das unsicher-desorgansierte/desorientierte Bindungsmuster postuliert [14]. Je belastender die kindlichen Erfahrungen sind, desto wahrscheinlicher ist die Ausbildung von einem der drei unsicheren Bindungsstile [15].

Die im Laufe der ersten Lebensjahre gesammelten Erfahrungen prägen den Bindungsstil bzw. auch die Ausformung bestimmter Selbst- und Fremdbilder [16]; diese neigen zwar zu einer gewissen Stabilität, allerdings zeigt die Studienlage auch, dass neue Beziehungserfahrungen - auch in unterschiedlichen (kulturellen) Kontexten - zu einer Verformung des ursprünglichen Bindungsstiles führen können [17]. Das Bindungskonzept besagt, dass auch im Erwachsenenalter Beziehungen eingegangen werden, da diese eine gewisse Bindungsfunktion erfüllen [18, 19]. Um die Qualität der Erwachsenenbindungen erheben zu können, kamen unterschiedliche - qualitative [20] sowie quantitative [19] - Methoden zum Einsatz. Während die quantitativen Erhebungsmethoden zunächst kategorial konzipiert wurden [19], haben sich mittlerweile dimensionale Messverfahren durchgesetzt. Die Anzahl der Dimensionen variiert dabei je nach Autor, wie beispielsweise im gut etablierten Modell der Erwachsenenbindung, dem Four-Category Attachment Measure (AAS4) von Bartholomew und Horowitz [21]. Die Autoren postulieren in Anlehnung an die Bindungstheorie sowohl positive als auch negative Selbst- und Fremd- bzw. Partnerbilder.

Der kulturellen Prägung der Bindungsstile kommt eine besondere Rolle $\mathrm{zu}$, da bedingt durch diese und die laufende Anpassung an den gesellschaftspolitischen Wandel, kontinuierliche Veränderung der Familienstrukturen zu beobachten sind [22]. In diesem $\mathrm{Zu}$ sammenhang scheinen die Begriffe Individualismus und Kollektivismus relevant [23]. Individualistisch geprägte Personen agieren primär ihrem persönlichen Wohl entsprechend, wodurch die Gemeinschaft, die wiederum dem Wohle des Kollektivisten dienlicher ist und dadurch dem Individuum gegenüber vorgezogen wird, hinten angestellt wird. Dabei vermittelt der Kern des Kollektivs individuelle Sicherheit. Der Kern einer Beziehung wird jedoch vom Individuum selbst geprägt [24, 25]. Die Dichotomie dieser beiden Begriffe wird häufig zur Unterscheidung zwischen westlichen und nicht-westlichen Kulturen herangezogen [26] und dient dazu, auf die Werte und Normen einer Kultur und die damit einhergehenden zwischenmenschlichen Beziehungen sowie den Familienzusammenhalt, sei es in der Kern- oder der Großfamilie, hinzuweisen [23]. Genau diese Normen und Werte bekommen insbesondere bei einer Migration einen besonderen Stellenwert [27]. So entwickelten beispielsweise die Türken durch die Migration nach Europa im Laufe der Zeit eine neue und eigene Immigrantenkultur in ihrer neuen Wahlheimat, im Rahmen derer sie sich weg vom System der Großfamilie hin zur Kernfamilie mit zurückgebliebener Verwandtschaft entwickelten [28]. Diese neue Kultur setzt sich aus den Traditionen der Herkunftskultur sowie aus ökonomischen, gesellschaftlichen und politischen Komponenten der Wahlkultur zusammen [29].

Seit den siebziger Jahren setzt sich die Wissenschaft auch vermehrt mit der Frage auseinander, ob Migration als pathogener bzw. Risikofaktor für psychische Erkrankungen gesehen werden kann. Diese konnte in mehreren empirischen Untersuchungen bestätigt werden [30]. Anhand der Prävalenzraten für psychische Erkrankungen, insbesondere der depressiven Störung, lässt sich nachweisen, dass Migranten häufiger als die österreichische Gastpopulationen unter diesen leiden [31-33].

Depressive Störungen zählen zur Kategorie der affektiven Störungen, da die primäre Symptomatik die 
Stimmungslage betrifft und als sekundäre Begleiterscheinungen körperliche Symptome sowie Störungen des Antriebs und der Psychomotorik auftreten [34, 35]. Die Zahlen zur Prävalenz variieren je nach Stichproben und Diagnosekriterien, wobei jedoch einige Quellen deckungsgleich von einer Lebenszeitprävalenz zwischen 15-20\% berichten [35-37]. Im Zusammenhang mit depressiven Störungen werden soziobiographische Faktoren, wie zum Beispiel Alter, Geschlecht, Ausbildung und Beruf, sowie die kulturellen Werte und Normen, das Verhalten, aber auch die Sprache als wichtige Einflussfaktoren für die Symptomäußerung genannt. So können die genannten Größen sich auf die subjektive Symptominterpretation, die Art der Belastungsbewältigung und die Hilfesuche auswirken. Außerdem wird die soziale Antwort auf die Belastung bzw. die gesellschaftliche Akzeptanz der Krankheit wesentlich vom kulturellen Hintergrund beeinflusst [38-40]. Eine Vergleichsstudie der depressiven Symptomatik von türkisch- vs. österreichstämmigen Patientinnen zeigte, dass türkische Migrantinnen im Vergleich zu Türkinnen im Herkunftsland und Österreicherinnen mehr somatische Beschwerden aufwiesen, was auf die bereits genannten Migrationseffekte zurückzuführen sei. Auch konnten signifikant unterschiedliche Profile der depressiven Symptomatik aufgedeckt werden, wobei beide türkischen Populationen mehr Somatisierungen vorwiesen [41].

Aus der aktuellen Studienlage leitet sich die Annahme ab, dass ein Unterschied zwischen depressiven und nicht depressiven Personen hinsichtlich ihrer Bindungsqualität besteht und dass dieser durch den kulturellen Hintergrund moderiert wird. In individualistisch eingestellten Kulturen, wie zum Beispiel der österreichischen, wird gemäß dieser Mutmaßung ein stärkeres Bedürfnis nach Unabhängigkeit und ein geringeres Ausmaß an Trennungsangst und Vermeidung erwartet, als dies in einer kollektivistisch orientierten Kultur, wie etwa der türkischen, der Fall ist. Hingegen erscheint es naheliegend, dass bei depressiven Personen mit einem kollektivistisch gefärbten Hintergrund die Trennungsangst sowie die Vermeidung als unsichere Komponenten des Bindungsverhaltens verstärkt werden, bei den individualistischen geprägten depressiven Personen hingegen - aufgrund der höheren sozialen Akzeptanz der Störung - lediglich der Wunsch nach Unabhängigkeit eingedämmt wird. Auf dieser Grundlage postulieren wir die Hypothese, dass eine Wechselwirkung von Kultur und depressiver Störung hinsichtlich der Bindungsqualität besteht. Die vorliegende Studie verfolgt den Zweck, diese Annahme zu prüfen.

\section{Methoden}

\section{Stichprobe}

In der vorliegenden Studie wurden drei Populationen, nämlich Türken im Herkunftsland, türkische Migran- ten in Österreich und Österreicher, unter Einschluss beider Geschlechter, untersucht. Dabei wurde jeweils eine gesunde Vergleichsgruppe (VG; $N=153$ ) und eine aktuell in einer depressiven Episode befindliche Patientengruppe (PG; $N=144)$ rekrutiert.

Die Studie wurde von der Ethikkommission der Medizinischen Universität Innsbruck im Vorfeld genehmigt. Die Rekrutierung der PG erfolgte im Untersuchungszeitraum von August 2012 bis Juni 2015 an der Universitätsklinik für Psychiatrie in Innsbruck, im Sonnenpark Lans (pro mente Tirol) und im Psychiatrischen Krankenhaus Manisa (Manisa Ruh Sağlığı ve Hastalıkları Hastanesi) in der Türkei. Einschlusskriterien dabei waren ein Alter zwischen 18 und 65 Jahren sowie eine vom Facharzt diagnostizierte aktuelle depressive Episode (F31, F32, F33 nach ICD-10 [42]) ohne psychotische Symptome. Ausschlusskriterien waren schwere körperliche Störungen oder kognitive Beeinträchtigungen, die die Studienteilnahme beeinträchtigt hätten. Die VG wurden mittels Schneeballsystem in Tirol und Izmir rekrutiert. Als Ausschlusskriterien wurden hier ebenfalls schwere körperliche Erkrankungen sowie psychische Störungen festgelegt, die mittels Eigenangaben der VG erfragt wurden. Das schriftliche Einverständnis mittels Unterschrift der Untersuchungsteilnehmer auf dem Aufklärungsbogen, der auf die Datenschutzrichtlinien hinwies und den Zweck der Studie erläuterte, wurde ebenso als Einschlusskriterium definiert.

\section{Erhebungsinstrumente}

Um die Bindungsqualität zu erheben, kam der von Griffin und Bartholomew [43] entwickelte Relationship Scales Questionnaire (RSQ) zum Einsatz. Dieser erfasst anhand einer fünfstufigen Likert-Skala ( 1 = nicht zutreffend, $5=$ sehr zutreffend) den Ausprägungsgrad jedes der von Bartholomew und Horowitz [21] definierten vier ${ }^{1}$ prototypischen Bindungsmuster (AAS4). Insgesamt enthält der Fragebogen 30 Einzelitems, zu denen auch jene der Adult Attachment Scale [44] zählen. Durch die Addition mehrerer Einzelitems können vier Skalenwerte gebildet werden. Diese werden in der deutschen Version des RSQ mit „Angst vor Trennung“, „Angst vor Nähe“, „Fehlendes Vertrauen“ und „Wunsch nach Unabhängigkeit“ tituliert [45]. In der vorliegenden Arbeit wurde auf die Berechnung der vier Bindungsstile gemäß AAS4 verzichtet, da dadurch ein Varianzverlust zu erwarten war. Stattdessen wurden die besagten Skalenwerte berücksichtigt. Die psychometrische Überprüfung der türkischen Version des RSQ erfolgte durch Sümer und Güngör [46]. 
Tab. 1 Beschreibung der Stichprobe nach Alter und Geschlecht

\begin{tabular}{|c|c|c|c|c|}
\hline \multirow[t]{2}{*}{ VG/PG } & \multicolumn{2}{|l|}{ Alter } & \multicolumn{2}{|l|}{ Geschlecht } \\
\hline & $N$ & $\mathrm{M}(\mathrm{SD})$ & $N$ weiblich (\%) & $N$ männlich (\%) \\
\hline Österreichische VG (A) & 49 & $30,81(11,91)$ & $20(40,8)$ & $29(59,2)^{C, F}$ \\
\hline Migrantische VG (B) & 53 & $33,92(8,98)$ & $30(56,6)$ & $23(43,4)$ \\
\hline Türkische VG (C) & 51 & 29,83 $(11,96)$ & $36(7,05)^{A}$ & $12(25,0)$ \\
\hline Österreichische PG (D) & 47 & $45,39^{\mathrm{A}, \mathrm{B}, \mathrm{C}, \mathrm{Fa}}(8,10)$ & $30(63,8)$ & $17(36,2)$ \\
\hline Migrantische PG (E) & 48 & $44,30^{\mathrm{A}, \mathrm{B}, \mathrm{C}, \mathrm{F}}(8,96)$ & $29(60,4)^{A}$ & $19(39,6)$ \\
\hline Türkische PG (F) & 49 & $36,45^{\mathrm{C}}(9,38)$ & $37(75,5)$ & $12(24,5)$ \\
\hline Gesamt & $297^{b}$ & $36,68(11,48)$ & $182^{b}(60,9)$ & $112^{b}(37,5)$ \\
\hline
\end{tabular}

\section{Statistische Analysen}

Die Berechnung der Ergebnisse erfolgte mittels des Statistikprogramms SPSS, Version 23 sowie mittels $\mathrm{G}^{*}$ Power. Um die sechs Untersuchungsgruppen im Vergleich aufzuzeigen, wurde neben der beschreibenden Statistik (M, SD) bei nominalskalierten Daten der Chi-Quadrat-Test mit anschließenden Z-Tests zur Berechnung der Gruppenunterschiede eingesetzt. Bei intervallskalierten Daten wurden zur Berechnung der Unterschiedshypothesen ein- und auch zweifaktorielle Varianzanalysen mit anschließendem SchefféTest in der post-hoc Analyse durchgeführt. Außerdem wurden Einzelgruppenvergleiche mittels T-Tests durchgeführt. Bei multiplem Testen erfolgte eine Alpha Adjustierung mittels Bonferroni Korrektur.

Zur Überprüfung der Teststärke wurde eine Posthoc-Poweranalyse bei einem Effekt mittlerer Stärke $(f=0,25)$ und einem Signifikanzniveau von $\alpha=0,05$ durchgeführt.

\section{Ergebnisse}

An der Untersuchung nahmen insgesamt 297 Personen teil. Dabei umfasste die Vergleichsgruppe 53 $(17,7 \%)$ Migranten, 51 (17,1\%) Türken und 49 (16,4\%) Österreicher. Die Patientengruppe setzte sich aus $49(16,4 \%)$ Türken, 48 (16,1\%) Migranten sowie 47 $(15,7 \%)$ Österreichern zusammen. Die demographischen Basisvariablen der einzelnen Gruppen sind in Tab. 1 dargestellt. Die Teststärke ergab die Kulturunterschiede und Interaktionseffekte betreffend einen Effekt von 97,7\%. Die Überprüfung der Unterschiede zwischen den Gesunden und Patienten zeigte einen Effekt von $99 \%$.

Die Altersstruktur der Teilnehmer zeigte signifikante Unterschiede, wobei vor allem die österreichische sowie migrantische PG, welche die zwei ältesten Altersdurchschnitte repräsentierten, sich signifikant $(p=0,01)$ von allen anderen Gruppen unterschieden. Die türkische VG stellte mit einem Altersdurchschnitt von 29,83 $(\mathrm{SD}=11,96)$ die jüngste Gruppe dar. Sowohl die türkische PG als auch die türkische VG wiesen signifikant $(p=0,00)$ mehr weibliche Teilnehmer auf als die österreichische VG.

Da aufgrund dieser Ergebnisse die Vergleichbarkeit der Gruppen in Frage gestellt war, wurde geprüft, ob das Alter, das Geschlecht, der Zivilstand, die Schulbildung und das Religionsbekenntnis einen Einfluss auf die abhängigen Variablen hatten. Diese Analyse wurde sowohl für die gesamte Untersuchungsgruppe als auch für die einzelnen Subgruppen durchgeführt. Es konnten keinen signifikanten Auswirkungen dieser Merkmale festgestellt werden. Es wurde außerdem für jedes dieser Merkmale einzeln geprüft, ob deren Berücksichtigung als Kovariate einen Einfluss auf die Ergebnisse der Varianzanalysen hatte. Auch diese Berechnungen führten zu keinen signifikanten Befunden. Das Ergebnis dieser Analyse spricht dafür, dass die im Folgenden berichteten Ergebnisse nicht auf Unterschiede zwischen den Untersuchungsgruppen in den demographischen Merkmalen zurückzuführen sind.

Nachfolgend werden lediglich die signifikanten Resultate des RSQ dargestellt. Nicht signifikante Ergebnisse werden ausschließlich aus Gründen der Nachvollziehbarkeit und Relevanz erwähnt. Insgesamt konnten kulturunabhängig signifikante Unterschiede zwischen der gesunden und depressiven Gesamtpopulation in den Skalen "Angst vor Trennung“ $(\mathrm{t}(280)=3,17 ; \quad p=0,00 ; \quad \mathrm{M}=3,55 ; \quad \mathrm{SD}=1,24), \quad$,Angst vor Nähe" ( $\mathrm{t}(290)=4,18 ; p=0,00 ; \mathrm{M}=3,66 ; \mathrm{SD}=1,29)$, „Fehlendes Vertrauen“ ( $\mathrm{t}(290)=5,07 ; p=0,00 ; \mathrm{M}=3,60$; $\mathrm{SD}=1,35)$ und ein tendenziell signifikanter Unterschied den „Wunsch nach Unabhängigkeit“ ( $\mathrm{t}(290)$ $=1,99 ; p=0,04 ; \mathrm{M}=4,68 ; \mathrm{SD}=1,30$ ) betreffend festgestellt werden. Im Vergleich der drei ethnischen Gesamtgruppen $(\mathrm{F}(2,289)=4,09 ; p=0,02 ; \mathrm{M}=3,60 ; 1,35)$ zeigten die Migranten ein signifikant höheres fehlendes Vertrauen als die Österreicher. Die österreichische Gruppe gab einen signifikant stärkeren Wunsch nach Unabhängigkeit $(\mathrm{F}(2,289)=18,78 ; p=0,00 ; \mathrm{M}=4,68$; $\mathrm{SD}=1,30$ ) an als die beiden türkischen Gruppen.

Bei einzelner Betrachtung der vier Skalen ergaben sich hinsichtlich der „Angst vor Trennung“ (Abb. 1) keine signifikanten interethnischen Unterschiede die Vergleichsgruppen betreffend. Die Patientengrup- 
Abb. 1 Angst vor Trennung im inter- und intrakulturellen Vergleich

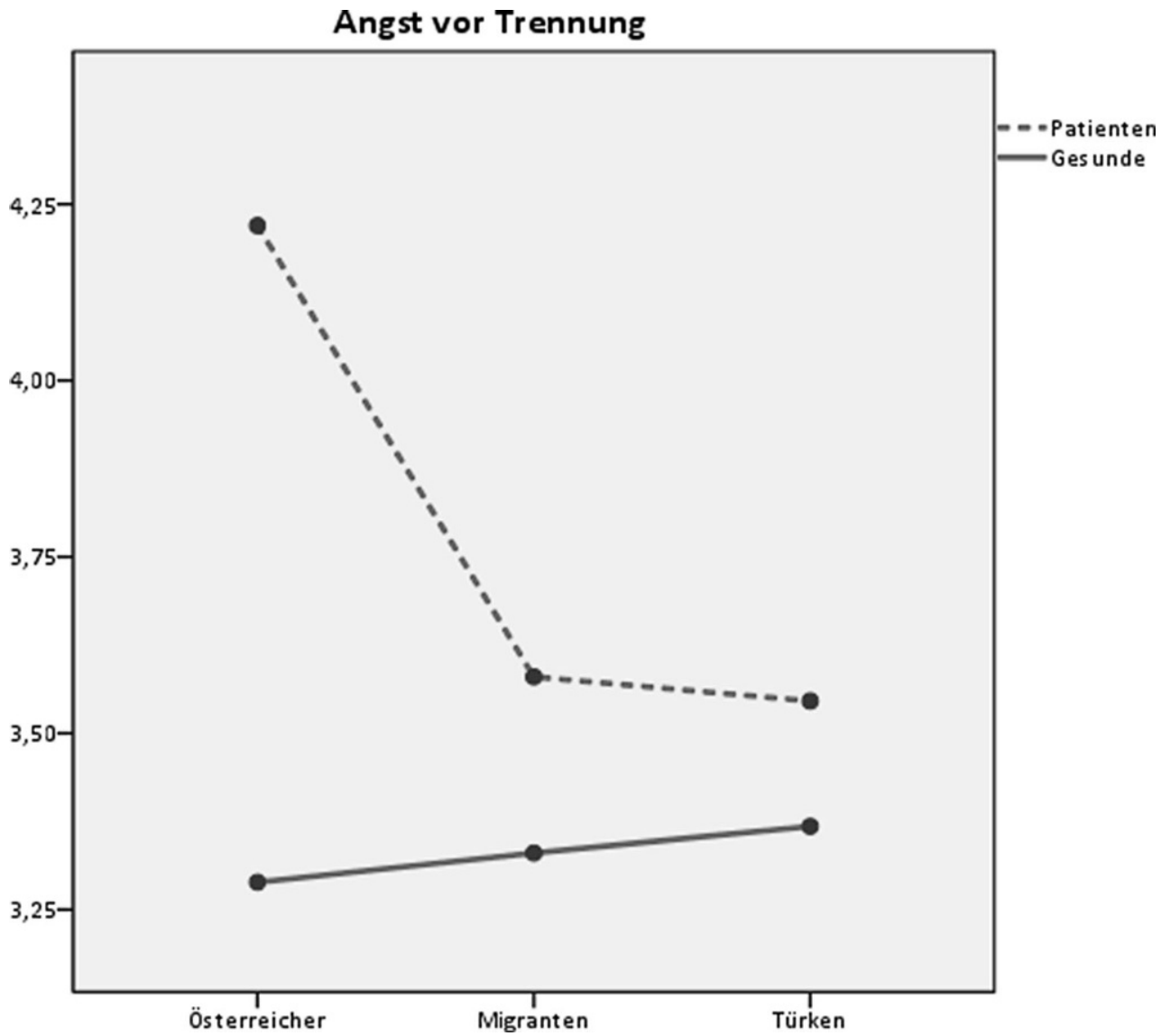

pen hingegen unterschieden sich signifikant $(\mathrm{F}(2$, 136) $=4,33 ; p=0,02 ; M=3,78 ; S D=1,28)$. Dabei zeigte die österreichische PG $(M=4,22 ; \mathrm{SD}=1,23)$ die größte Angst vor Trennung und unterschied sich somit signifikant $(p=0,03)$ von der türkischen $(\mathrm{M}=3,55$; $\mathrm{SD}=1,15)$ und tendenziell signifikant $(p=0,05)$ von der migrantischen PG $(\mathrm{M}=3,44 ; \mathrm{SD}=1,32)$. Bei den Österreichern zeigten sich des Weiteren hochsignifikante intrakulturelle Unterschiede $(\mathrm{t}(94)=3,86$; $p=0,00)$ zwischen den Patienten $(\mathrm{M}=4,22 ; \mathrm{SD}=1,23)$ und den Gesunden $(M=3,29 ; \mathrm{SD}=1,14)$. Es gab einen tendenziellen Interaktionseffekt $\quad(\mathrm{F}(2,286)=2,87$; $p=0,05 ; \mathrm{M}=3,55 ; \mathrm{SD}=1,24)$ zwischen der Kultur und der Störung hinsichtlich der Angst vor Trennung, was einen Hinweis auf die vermutete Wechselwirkung zwischen der Kultur und der depressiven Störung darstellt.

Die „Angst vor Nähe“ (Abb. 2) zeigte signifikante Unterschiede zwischen den drei Vergleichsgruppen $(\mathrm{F}(2,150)=3,84, p=0,03 ; \mathrm{M}=3,34 ; \mathrm{SD}=1,10)$ und war bei der türkischen VG $(\mathrm{M}=3,58 ; \mathrm{SD}=1,28)$ am stärksten ausgeprägt. Diese unterschied sich signifikant $(p=0,03)$ von der österreichischen VG $(\mathrm{M}=3,01$; $\mathrm{SD}=0,87$ ). Die Patientengruppen zeigten keine signifikanten Unterschiede untereinander, wohingegen signifikante intrakulturelle Unterschiede wiederum bei den Österreichern $(\mathrm{t}(80)=4,10 ; p=0,00$; PG: $\mathrm{M}=3,94$; $\mathrm{SD}=1,30$; VG: $\mathrm{M}=3,01 ; \mathrm{SD}=0,87)$ sowie bei den $\mathrm{Mi}-$ granten $(\mathrm{t}(71)=2,97 ; p=0,00 ; \mathrm{PG}: \mathrm{M}=4,24 ; \mathrm{SD}=1,54$; VG: $M=3,56$; $S D=1,04)$ zu sehen waren. Erneut wurde ein tendenzieller Interaktionseffekt zwischen Kultur und Störung $(\mathrm{F}(2,286)=2,89 ; p=0,05 ; \mathrm{M}=3,64$; $\mathrm{SD}=1,29$ ) in Bezug auf die Bindungsdimension festgestellt.

Hinsichtlich des „Fehlenden Vertrauens“ (Abb. 3) zeigten sich signifikante Differenzen bei den Vergleichsgruppen ( $\mathrm{F}=6,90 ; p=0,00 ; \mathrm{M}=3,24 ; \mathrm{SD}=1,21)$. Die österreichische VG $(\mathrm{M}=2,74 ; \mathrm{SD}=1,12)$, welche die geringste Ausprägung in dieser Skala aufwies, unterschied sich signifikant sowohl $(p=0,00)$ von der migrantischen $(\mathrm{M}=3,56 ; \mathrm{SD}=1,18)$ als auch $(p=0,02)$ von der türkischen VG $(\mathrm{M}=3,38 ; \mathrm{SD}=1,20)$. Wie in der vorangegangenen Skala zeigten sich auch hier keine signifikanten Unterschiede zwischen den einzelnen Patientengruppen, jedoch waren intrakulturelle Unterschiede bei den Österreichern $(\mathrm{t}(94)=5,79 ; p=0,00$; PG: $\mathrm{M}=4,04 ; \mathrm{SD}=1,08 ; \mathrm{VG}: \mathrm{M}=2,74 ; \mathrm{SD}=1,12)$ sowie bei den Migranten $(\mathrm{t}(76)=2,70 ; p=0,01) \mathrm{zu}$ verzeichnen. Es waren außerdem signifikante Interaktionseffekte $(\mathrm{F}(2,286)=3,93 ; p=0,02 ; \mathrm{M}=3,60 ; \mathrm{SD}=1,35)$ zwischen Kultur und Störung hinsichtlich dem fehlenden Vertrauen feststellbar.

Den „Wunsch nach Unabhängigkeit“ (Abb. 4) betreffend zeigten sich signifikante Unterschiede zwischen den Vergleichsgruppen $(\mathrm{F}(2,150)=9,78$; $p=0,00 ; \mathrm{M}=4,54 ; \mathrm{SD}=1,10$ ), insbesondere zwischen 
Abb. 2 Angst vor Nähe im inter- und intrakulturellen Vergleich

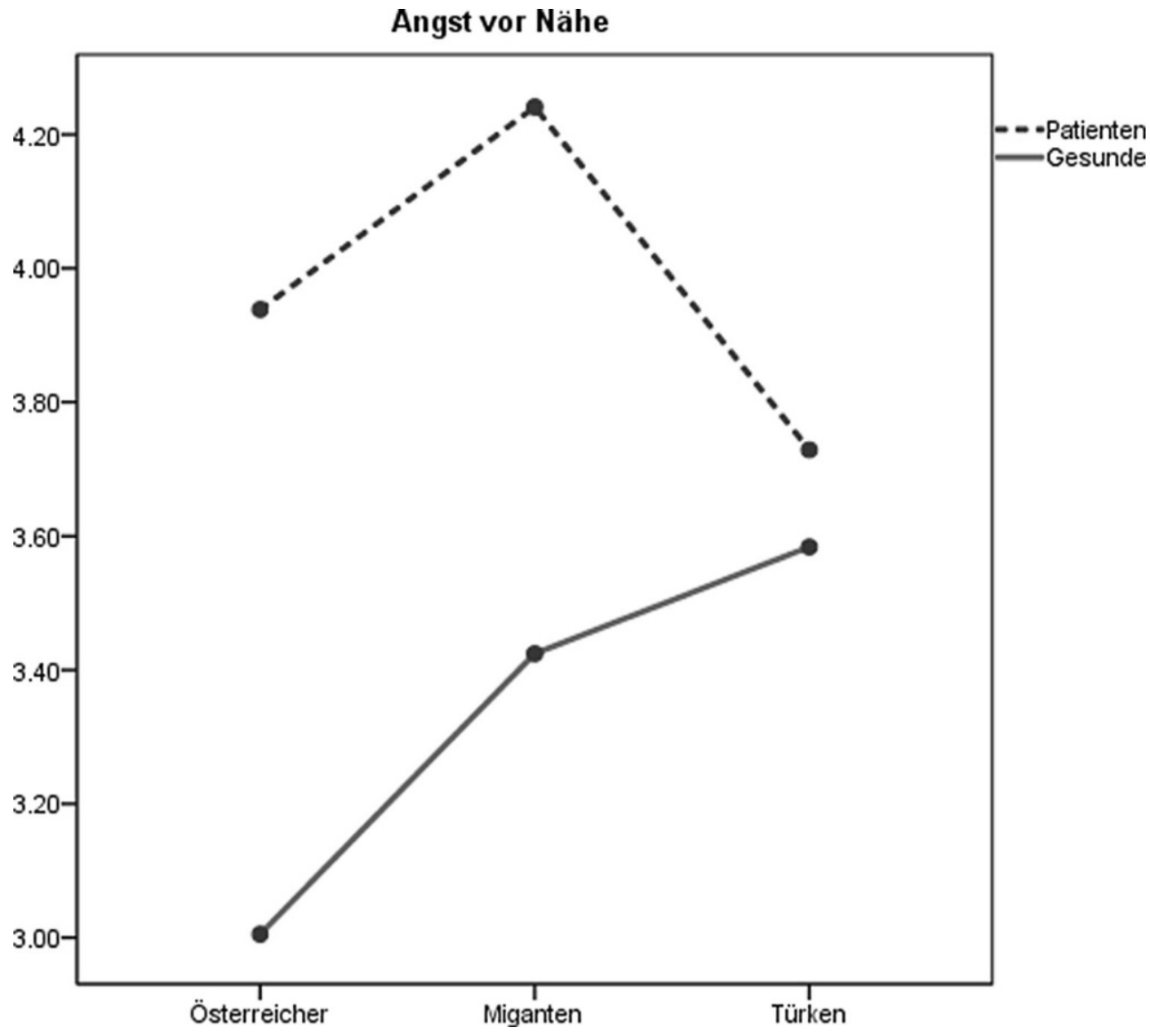

der österreichischen VG $(M=5,08 ; \mathrm{SD}=1,01)$, welche die höchsten Werte aufwies, und der migrantischen $(\mathrm{M}=4,26 ; \mathrm{SD}=1,10)$ VG $(p=0,00)$ sowie der österreichischen und der türkischen $(\mathrm{M}=4,32 ; \mathrm{SD}=1,00)$ VG $(p=0,00)$. Auch in der PG zeigte sich ein vergleichbar signifikantes Bild $(\mathrm{F}(2,136)=9,20 ; p=0,00$; $\mathrm{M}=4,84 ; \mathrm{SD}=1,48)$. Es konnten keine intrakulturellen Unterschiede oder Interaktionseffekte hinsichtlich der Kultur und der Störung festgestellt werden.

\section{Diskussion}

Die Ergebnisse der vorliegenden Studie erwiesen sich als insgesamt erwartungskonform, da sowohl intraals auch interkulturelle Unterschiede festgestellt werden konnten. Im interkulturellen Gesamtvergleich der drei Ethnien lagen die signifikanten Unterschiede zwischen den Österreichern und den beiden türkischstämmigen Gruppen, was aufgrund der unterschiedlichen kulturellen Hintergründe nachvollziehbar erscheint und mit der gängigen Theorie im Einklang steht [23, 24]. Dieser zufolge weisen die Österreicher ein „independent self“ auf, wonach sie ihre eigenen, auch beziehungsbezogenen Interessen vorrangig behandeln. Die türkischstämmigen Personen hingegen verkörpern das „interdependent self“, wonach das Kollektiv und die sozialen Erwartungen über die eigene Person und die damit einhergehenden be- ziehungsrelevanten Entscheidungen gestellt werden [24].

Erwartungsgemäß zeigten sich - sowohl bei den PG als auch bei den VG - interkulturelle Unterschiede in den verschiedenen Bindungsskalen. Die türkische VG offenbarte im Gegensatz zu der österreichischen VG die größte „Angst vor Nähe“ und die höchste Ausprägung an „Fehlendem Vertrauen“. Auch die migrantische VG wies gegenüber der österreichischen VG höhere Werte an „Fehlendem Vertrauen“ auf. Diese wiederum zeigte die höchste Ausprägung im „Wunsch nach Unabhängigkeit“. Insgesamt ist die österreichische VG somit als sicherer gebunden [43] einzustufen. Entgegen der ursprünglichen Annahme zeigte die österreichische Patientengruppe bei einer interkulturellen Betrachtung im Vergleich $\mathrm{zu}$ den beiden türkischen PG höhere Ausprägungen in den Skalen „Angst vor Trennung“ sowie „Wunsch nach Unabhängigkeit“. Die Vergleichsgruppen waren insgesamt im Vergleich zu den Patientengruppen in allen Parametern als sicherer einzustufen, da sie durchwegs niedrigere Skalenwerte aufwiesen; diese Ergebnisse decken sich ebenfalls mit der aktuellen Studienlage [47, 48], wonach depressive Störungen u. a. selbstwertreduzierende Effekte haben und neben einem sozialen Rückzug mit Angst vor Zurückweisung sowie pessimistischen Beziehungserwartungen einhergehen können. 
Abb. 3 Fehlendes Vertrauen im inter- und intrakulturellen Vergleich

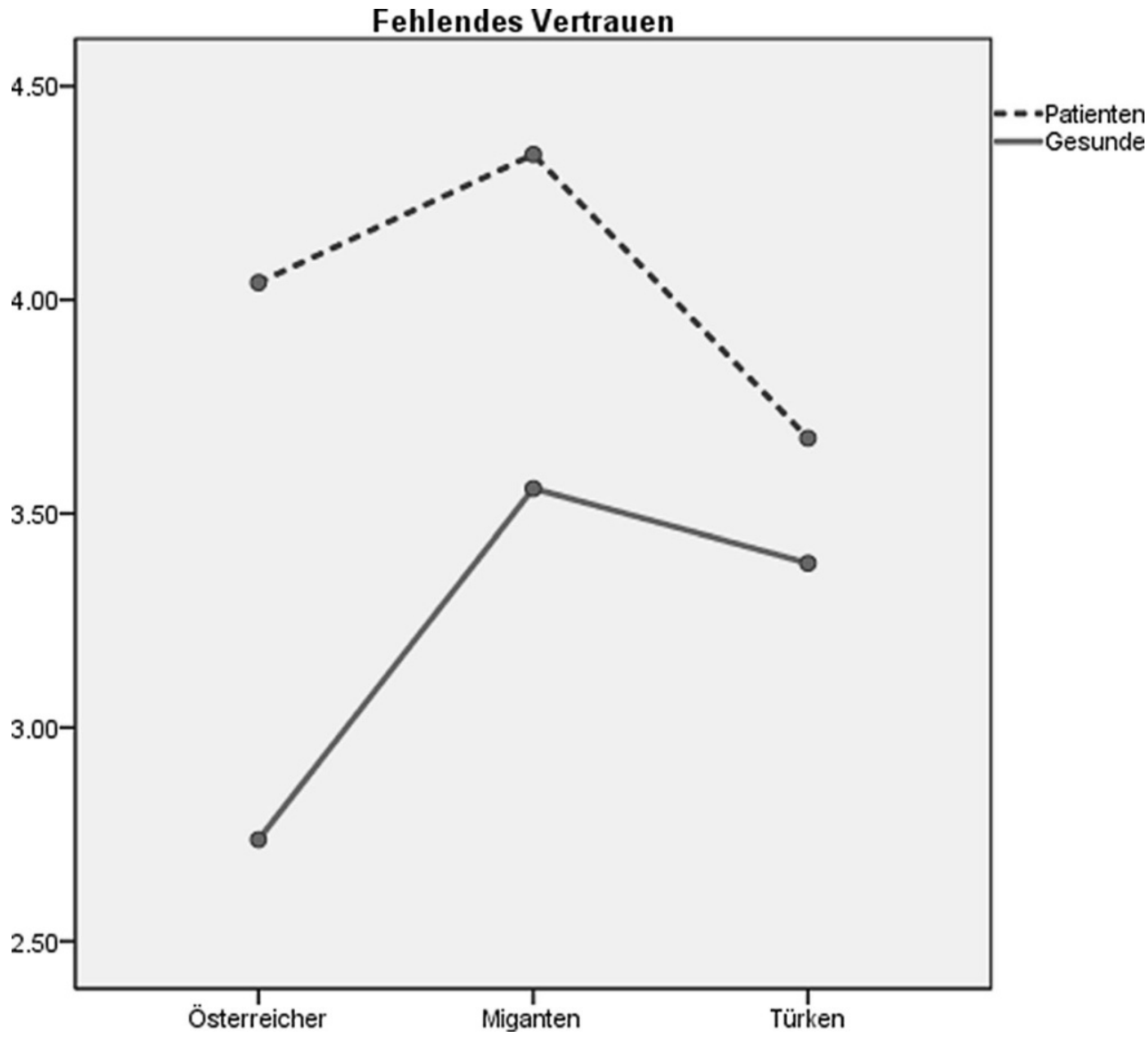

Im Vergleich der intrakulturellen Unterschiede der drei ethnischen Gruppen zeigten sich teilweise unerwartete Ergebnisse. Bei der österreichischen PG waren die „Ängste vor Trennung und Nähe“ sowie das „Fehlende Vertrauen“ stärker ausgeprägt als bei der VG. Auch zwischen den gesunden und depressiven Migranten fanden sich vergleichbare Unterschiede die „Angst vor Nähe“ sowie das „Fehlende Vertrauen“ betreffend. Die türkische PG sowie VG hingegen zeigten keine signifikanten Unterschiede in Bezug auf ihre Bindungsorientierung.

Diese Ergebnisse lassen den Schluss zu, dass das depressive Krankheitsbild und die Kultur in Hinblick auf die Bindungsqualität miteinander interagieren bzw. in einer Wechselwirkung stehen. Dies kann aus den Interaktionseffekten, die hinsichtlich dem „Fehlenden Vertrauen“ signifikant, in Bezug auf die „Ängste vor Nähe und Trennung" tendenziell signifikant waren, abgeleitet werden. Bar einer vergleichbaren theoretischen Grundlage muss die Interpretation der Interaktion spekulativ und auf den bereits in der Einleitung dargelegten, allgemeinen Überlegungen basierend erfolgen.

Die Betrachtung des kulturellen Hintergrundes in Zusammenhang mit individualistischen und kollektivistischen [23, 24] Familien- und Beziehungsstrukturen $[9,10,12]$ erscheint für die Diskussion der Ergebnisse essenziell. So versuchen die Türken im
Herkunftsland aufgrund ihrer der Gesellschaft den Vorrang einräumenden Mentalität [26] genau dieser und deren Vorstellungen - auch was Beziehungen anbelangt - gerecht zu werden, um sich der gesellschaftlichen Akzeptanz sicher sein zu können. Einerseits scheint das eingeräumte Vorrecht des Kollektivs zu Einbußen in der Bindungssicherheit bei gesunden Personen zu führen, doch andererseits legen die Ergebnisse im intrakulturellen Vergleich eine Funktion als Auffangnetz im Fall einer depressiven Störung nahe. In der österreichischen, „westlichen“ [26] Mentalität, wird das Wohlergehen der eigenen Person dem Wohl Anderer vorgezogen [24]. Dies scheint mit positiv gefärbten Beziehungen im Erwachsenenalter in Verbindung zu stehen, nachdem die österreichischen VG die höchste Bindungsqualität zeigten. Doch die Ergebnisse legen den Schluss nahe, dass durch den zunehmenden Individualismus sich das soziale Gefüge auf - zumeist - die Kernfamilie [49] reduziert, wodurch es im Fall einer depressiven Erkrankung zu einer Störungsanfälligkeit der Bindungsorientierung kommen könnte. Die Ergebnisse der Migranten deuten auf eine Verbundenheit mit ihrer Herkunftskultur [28] sowie den Versuch, dieser mit ihren Normen und Werten - inklusive den internalisierten Familien- und Beziehungsstrukturen - gerecht $\mathrm{zu}$ werden, hin. Dennoch sind Hinweise auf eine gewisse Entfernung von der Heimatkultur hin zur Gastpopulation 
Abb. 4 Wunsch nach Unabhängigkeit im inter- und intrakulturellen Vergleich

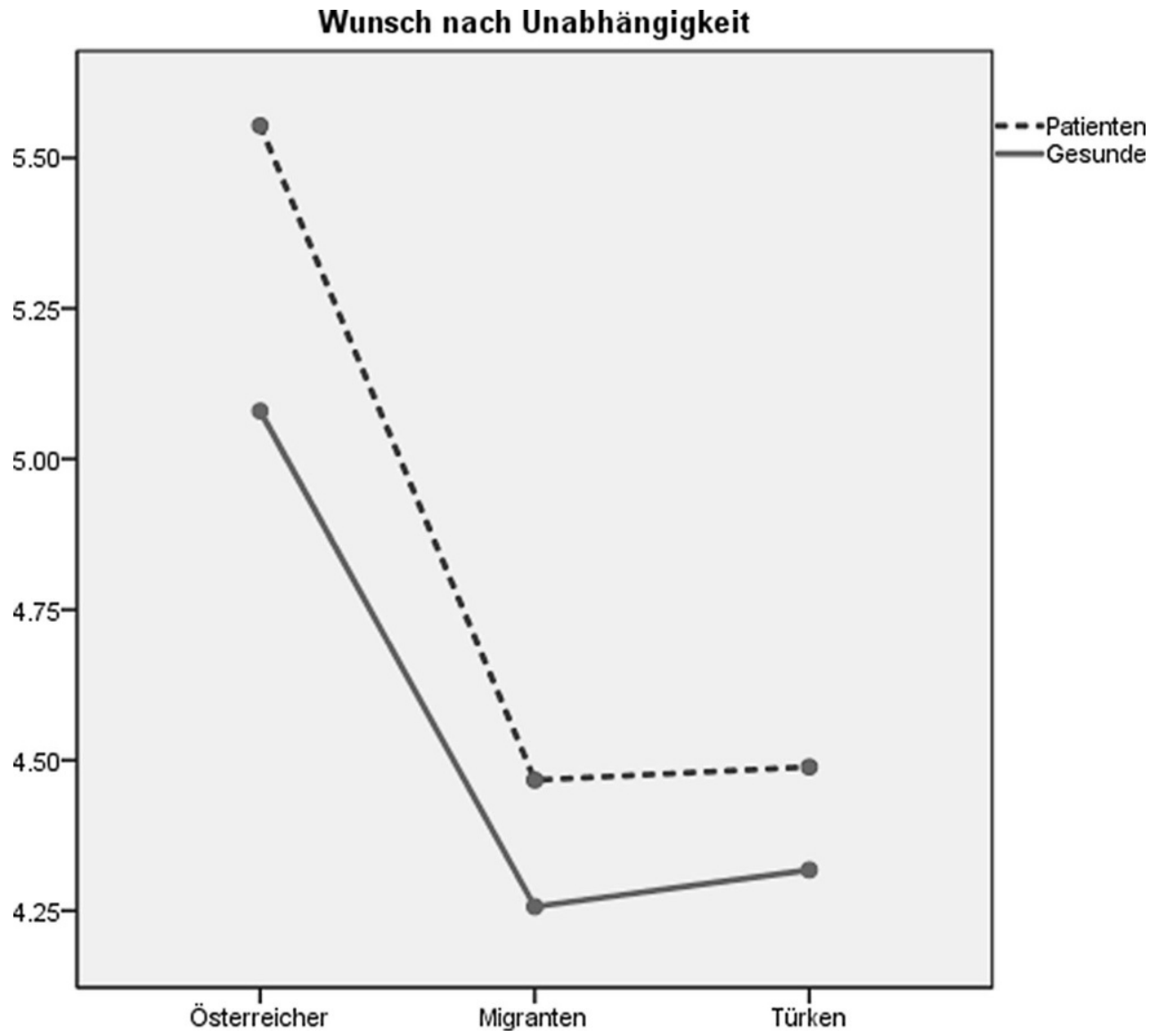

sichtbar, die auch anhand der teilweise vergleichbaren Ergebnisse der migrantischen sowie österreichischen Patientengruppen unterstrichen werden können. Trotz der fehlenden Signifikanzen zeigten sich deutliche Unterschiede zwischen den Migranten und ihrer Herkunftskultur, die für eine Entwicklung einer Immigrantenkultur [29] oder aber auch eine fortschreitende Assimilation [50] sprechen könnten.

\section{Limitation, Stärken und Ausblick}

In der vorliegenden Arbeit wurden inter- und intrakulturelle Unterschiede dreier ethnischer Patienten- und Vergleichsgruppen in Bezug auf deren Bindungsqualität untersucht. Dabei konnte eine ausreichend große Stichprobe eingeschlossen werden, um einen repräsentativen Querschnitt der drei Kulturen erfassen zu können. Es konnten Instrumente verwendet werden, die sowohl im Deutschen wie im Türkischen validiert sind.

Auch wenn versucht wurde, den Einfluss der demographischen Merkmale sorgfältig zu prüfen, müssen die Unterschiede in der Altersstruktur und die unterschiedliche Geschlechterverteilung als Limitation erwähnt werden. Außerdem gilt in der Bindungsforschung eine quantitative Erhebung mittels Fragebögen als Einschränkung. Mittels Selbstauskünften in Fragebögen können psychodynamische und biogra- fische Aspekte sicherlich nicht erfasst werden. Interviewverfahren, wie das Adult Attachment Interview [18], die Verarbeitung früher Bindungserfahrungen erfassen, können gegebenenfalls Einblicke in den Ursprung depressiver Störungen vermitteln. Da ein Kulturvergleich nur anhand umfangreicherer Stichproben möglich ist, wurde in der vorliegenden Studie auf quantitative Verfahren zurückgegriffen.

Eine weitere Limitierung der Studie stellt die Tatsache dar, dass die Diagnosen der Patienten nicht mittels strukturierter Fragebögen, sondern klinisch-fachärztlich, wenn auch nach den Kriterien des ICD-10, gestellt wurden. Aus logistischen Gründen war eine gemeinsame Schulung der involvierten Ärzte im Sinne eines Interrater-Trainings nicht durchführbar, sodass ein möglicher kultureller Einfluss diesbezüglich nicht ausgeschlossen werden kann.

Bei der aktuellen Studie handelt es sich um eine Querschnittuntersuchung an Gruppen, wodurch auch keine gesicherten Aussagen über die Wechselwirkung beziehungsweise kausale Zusammenhänge der Depression mit den Bindungsstilen getroffen werden können. In weiterer Folge interessant wäre eine Längsschnittuntersuchung, in dessen Rahmen nicht nur die ätiologischen Gesichtspunkte der depressiven Erkrankung im interkulturellen Vergleich erhoben, sondern auch eine mögliche Veränderung der Bindungsstile 
möglicherweise durch den Einfluss der depressiven Erkrankung beleuchtet werden könnten.

Die für die aktuelle Studie rekrutierten Personen stammen aus städtischen Regionen. Interessante Aspekte könnten für zukünftige Studien auch Vergleiche zwischen dörflichen und städtischen Regionen liefern. Auch wäre ein Vergleich anderer ethnischer Gruppierungen, Patientengruppen mit anderen Diagnosen oder die Fokussierung auf den Einfluss unterschiedlicher Religionen und Religiosität denkbar. Im interkulturellen Kontext lässt sich hier ein vielversprechendes Forschungsfeld, auch was unterschiedliche therapeutische Schwerpunktsetzungen bei depressiven Patienten unterschiedlicher Kulturen betrifft, aufspannen.

Funding Open access funding provided by University of Innsbruck and Medical University of Innsbruck.

\section{Einhaltung ethischer Richtlinien}

Interessenkonflikt E. Gündüz, B.H. Juen, W. Geser, A. Ayer und E.A. Deisenhammer geben an, dass kein Interessenkonflikt besteht.

Ethische Standards Alle beschriebenen Untersuchungen am Menschen wurden mit Zustimmung der zuständigen EthikKommission, im Einklang mit nationalem Recht sowie gemäß der Deklaration von Helsinki von 1975 (in der aktuellen, überarbeiteten Fassung) durchgeführt. Von allen beteiligten Patienten liegt eine Einverständniserklärung vor.

Open Access Dieser Artikel wird unter der Creative Commons Namensnennung 4.0 International Lizenz (http:// creativecommons.org/licenses/by/4.0/deed.de) veröffentlicht, welche die Nutzung, Vervielfältigung, Bearbeitung, Verbreitung und Wiedergabe in jeglichem Medium und Format erlaubt, sofern Sie den/die ursprünglichen Autor(en) und die Quelle ordnungsgemäß nennen, einen Link zur Creative Commons Lizenz beifügen und angeben, ob Änderungen vorgenommen wurden.

\section{Literatur}

1. Bowlby J. Bindung und Verlust. Bd. 1. München, Basel: E. Reinhardt;2006.

2. Steele M. Bindung, reale Erfahrung und mentale Repräsentation. In: Green V, Hrsg. Emotionale Entwicklung in Psychoanalyse, Bindungstheorie und Neurowissenschaften: Theoretische Konzepte und Behandlungspraxis. Frankfurt a.M.: Brandes \&Apsel; 2005. S. 115-40.

3. Asendorpf J, Banse R. Psychologie der Beziehung. Aus dem Programm Huber : Psychologie-Lehrbuch. Bern: Huber; 2000.

4. Holmes J. John Bowlby und die Bindungstheorie. 2. Aufl. München:Ernst Reinhardt; 2006.

5. van den Boom DC. Do first-year intervention effects endure? Follow-up during Toddlerhood of a sample of Dutch irritable infants. Child Dev. 1995;66(6):1798.

6. Fox NA, Kimmerly NL, Schafer WD. Attachment to mother/attachment to father: a meta-analysis. Child Dev. 1991;62(1):210.

7. Schuengel C, Bakermans-Kranenburg MJ, van Ijzendoorn MH. Frightening maternal behavior linking unresolved loss and disorganized infant attachment. JConsultClin Psychol. 1999;67(1):54-63.

8. Thompson RA. Early sociopersonality development. In: Eisenberg N, Hrsg. Handbook of child psychology. 5. Aufl. New York:Wiley; 1998. S. 25-104.

9. Cassibba R, Sette G, Bakermans-Kranenburg MJ, et al. Attachment the Italian Way. Eur Psychol. 2013;18(1):47-58.

10. Sagi A, Lamb ME, Lewkowicz KS, et al. Security of infantmother, -father, and -metapelet attachments among kibbutz-reared Israeli children. Monogr Soc Res Child Dev. 1985;50(209):257-75.

11. Zevalkink J, Riksen-Walraven JM, Van Lieshout CFM. Attachment in the Indonesian caregiving context. Soc Dev. 1999;8(1):21-40.

12. Rothbaum F, Weisz J, Pott M, et al. Deeper into attachment and culture. Am Psychol. 2000;56(10):827-9.

13. Ainsworth M-DS. Infant-mother attachment. Am Psychol. 1979;34(10):932-7.

14. Main M, Hesse E, Hesse S. Attachment theory and research: overview with suggested applications to child custody. Fam Court Rev. 2011;49(3):426-63.

15. Brisch KH. Bindungsstörungen: Von der Bindungstheorie zurTherapie. 9. Aufl. Stuttgart: Klett-Cotta; 2009.

16. Bowlby J. Bindung als sichere Basis: Grundlagen und Anwendung der Bindungstheorie. München, Basel: E. Reinhardt; 2008.

17. Waters E, Merrick S, Treboux D, et al. Attachment security in infancy and early adulthood: a twenty-year longitudinal study. Child Dev.2000;71(3):684-9.

18. Main M, Kaplan N, CassidyJ. Security in infancy, childhood, and adulthood: a move to the level of representation. Monogr Soc Res Child Dev. 1985;50(1/2):66.

19. Hazan C, Shaver P. Romantic love conceptualized as an attachment process. J Pers Soc Psychol. 1987;52(3):511-24.

20. George C, Kaplan N, Main M. Kapitel 15: Adult Attachment Interview. In: Gloger-Tippelt G, Hrsg. Bindung im Erwachsenenalter:Ein Handbuch für Forschung und Praxis. 2. Aufl. Bern:Huber; 2012. S. 419-40.

21. Bartholomew K, Horowitz LM. Attachment styles among young adults: a test of a four-category model. J Pers Soc Psychol. 1991;61(2):226-44.

22. Mitterauer M, Sieder R. Vom Patriarchat zur Partnerschaft: Zum Strukturwandel der Familie. 4. Aufl. Beck'sche Reihe, BsR. 158. München: C.H.Beck; 1991.

23. Triandis HC. Individualism \& collectivism. New directions in social psychology. Boulder:Westview Press; 1995.

24. Markus HR, Kitayama S. Culture and the self: Implications for cognition, emotion, and motivation. Psychol Rev. 1991;98(2):224-53.

25. Wermke M, Kraif U. Duden online. 2013. http://www. duden.de/.Zugegriffen:2.Febr. 2015.

26. Keller H, Eckensberger LH. Kultur und Entwicklung. In: Keller H, Hrsg. Lehrbuch Entwicklungspsychologie. 1. Aufl. Bern:Huber; 1998. S. 57-96.

27. Haas AW. Türkische Volksfrömmigkeit. Beiträge zur Ausländerarbeit, Bd. 9. Frankfurt am Main: Otto Lembeck; 1986.

28. Pasero U. Familienkonflikte in der Migration: Eine rechtssoziologische Studie anhand von Gerichtsakten. DUV Sozialwissenschaft. Wiesbaden:Dt. Univ.-Verl.; 1990.

29. Essinger H, Kula OB. Pädagogik als interkultureller Prozess: Beiträge zu einer Theorie interkultureller Pädagogik. Theorie, [1. Felsberg: Migro-Verl; 1987.

30. Assion H-J. Migration und psychische Krankheit. In: Assion H-J, Hrsg. Migration und seelische Gesundheit. Mit 13 Abbildungen und 22 Tabellen. Heidelberg: Springer; 2005. S. 133-44. 
31. Koch E, Hartkamp N, Siefen RG, et al. Patienten mit Migrationshintergrund in stationär-psychiatrischen Einrichtungen. Pilotstudie der Arbeitsgruppe „Psychiatrie und Migration“ der Bundesdirektorenkonferenz. Nervenarzt. 2008;79(3):328-39.

32. Merbach M, Wittig U, Brähler E. Angst und Depression polnischer und vietnamesischer MigrantInnen in Leipzig unter besonderer Berücksichtigung ihres Eingliederungsprozesses. Psychother Psychosom Med Psychol. 2008;58(3-4):146-54.

33. Levecque K, Lodewyckx I, Bracke P. Psychological distress, depression and generalised anxiety in Turkish and Moroccan immigrants in Belgium: a general population study. Soc Psychiatry Psychiatr Epidemiol. 2009;44(3):188-97.

34. Wunn E. Basics Psychiatrie. 1. Aufl. München: Elsevier, Urban \& Fischer; 2006

35. Arolt V, Pauli-Pott U, Reimer C, et al. Basiswissen Psychiatrie und Psychotherapie. Mit 63 Fallbeispielen und 13 Tabellen. 6. Aufl. Heidelberg: Springer; 2007.

36. Grabe HJ, Mahler J, Becker M. Frühe Traumatisierung und affektive Störungen. In: Spitzer C, Grabe HJ, Hrsg. Kindesmisshandlung: Psychische und körperliche Folgen im Erwachsenenalter. Stuttgart:Kohlhammer;2013.S. 146-60.

37. Rebhandl E, Stuppäck C, Brettleckner M-E, et al. Management der Depression in der Allgemeinmedizinischen Praxis: Konsensus-Meeting und -Statement unter der Ägide der ÖGAM. 2010. http://www.oegam. at/wissenschaft-publikationen/konsensus/2010/?no cache $=1 \&$ tx_drblob_pi1\%5BdownloadUid\%5D $=108 . \mathrm{Zu}-$ gegriffen:3. Febr. 2016.

38. Kirmayer LJ. Cultural variations in the clinical presentation of depression and anxiety: implications for diagnosis and treatment. JClin Psychiatry. 2001;62(suppl 13):22-8.

39. Özmen E, Ögel K, Boratav C, et al. Depresyon ile İlgili Bilgi ve Tutumlar: İstanbul Örneği. Turk Psikiyatri Derg. 2003;14(2):89-100.

40. Haasen C, Kleinmeier E, Yagdiran O. Kulturelle Aspekte bei der Diagnostik psychischer Störungen. In: Assion H-J, Hrsg. Migration und seelische Gesundheit. Mit 13 Abbildungen und 22 Tabellen. Heidelberg: Springer; 2005. S. 145-55.

41. Deisenhammer EA, Coban-Başaran M, Mantar A, et al. Ethnic and migrational impact on the clinical manifes- tation of depression. Soc Psychiatry Psychiatr Epidemiol. 2012;47(7):1121-9.

42. Dilling H, Mombour W, Schmidt MH, Hrsg. Internationale Klassifikation psychischer Störungen: ICD-10 Kapitel V (F): Klinisch-diagnostische Leitlinien. 8. Aufl. Bern: Huber; 2011. unter Berücksichtigung der Änderungen entsprechend ICD-10-GM.

43. Griffin DW, Bartholomew K. Models of the self and other: Fundamental dimensions underlying measures of adult attachment. J Pers Soc Psychol. 1994;67 (3):430-45.

44. Collins NL, Read SJ. Adult attachment, working models, and relationship quality in dating couples. J Pers Soc Psychol. 1990;58(4):644-63.

45. Steffanowski M, Oppl J, Meyerberg J, et al. Empirische Ergebnisse: Psychometrische Überprüfung einer deutschsprachigen Version des Relationship Scales Questionaire (RSQ). 2001. https://www.researchgate.net/ publication/248150720_Psychometrische_Uberprufung einer_deutschsprachigen_Version_des_Relationship_ Scales_Questionaire_RSQ.Zugegriffen:7.März2016.

46. Sümer N, Güngör D. Yetiskin baglanma stilleri ölcektlerinin Türk örneklemi üzerinde psikometrik degerlendirmesi ve kültürlerarasi bir karsilastirma. Türk Psikol Derg. 1999;14:71-106.

47. WHO. Programme on mental health: WHOQOL User Manual. Division of mental health and prevention of substance abuse. 1998. http://apps.who.int/iris/bitstream/ 10665/77932/1/WHO_HIS_HSI_Rev.2012.03_eng.pdf. Zugegriffen:21.Febr.2016.

48. Taminiau-Bloem EF, Visser MRM, Tishelman C, et al. Somatically ill persons' self-nominated quality of life domains: review of the literature and guidelines for future studies. Qual Life Res.2010;19(2):253-91.

49. Rathmayer B. Allein im Schlaraffenland? Zur Lebensrealität von Kindern in der Konsumgesellschaft. In: Romeike G, Imelmann H, Hrsg. Hilfen für Kinder: Konzepte und Praxiserfahrungen für Prävention, Beratung und Therapie. Weinheim, München: Juventa; 1999. S. 19-45. eine Veröffentlichung der Bundeskonferenz für Erziehungsberatung e. V.

50. BerryJW.Immigration, Acculturation, and Adaptation. Appl Psychol. 1997;46(1):5-34. 